For personal use only. Not to be reproduced without permission of the publisher (editorial@gabi-journal.net).

\section{Waiver of consent for retrospective chart review studies}

Professor Philip D Walson, MD

The importance of obtaining IRB/EC consent for waiver of consent before embarking on retrospective chart review studies was highlighted using the recent case of a manuscript submitted to GaBI Journal.

Keywords: Chart review, consent, retrospective, waiver

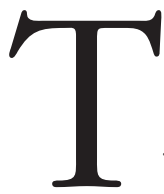

his commentary was prompted by a recent experience with a manuscript submitted to $G a B I$ Journal but then withdrawn.

The authors of this manuscript conducted a retrospective chart review of patients who had undergone cardiac stent placements at two hospitals in Greece to investigate whether there were any statistically significant differences in outcomes when patients were treated with either brand-name or generic versions of aspirin and clopidogrel. Peer review of the manuscript revealed that there was no statement as to whether the study had been approved by the local institutional review board/ ethics committee (IRB/EC) or any mention of whether either informed consent had been obtained or if waiver of consent was approved by the IRBs/ECs at the two involved hospitals. When questioned about this, the authors responded by stating that in their opinion there was no requirement for consent since the study involved only a retrospective chart review of anonymous patient data. On further questioning they admitted that the study had never been submitted to either hospital's IRB/EC for review or for permission to waive consent. In an attempt to publish the study, the authors were then asked to request a letter from their IRBs/ECs stating that, based on the local guidelines, that they agreed with the authors that no consent was required. The authors replied by claiming that since the study had been done more than two years prior to submission that it was not possible to get such a statement from the IRB and as a result they unfortunately simply withdrew the manuscript.

This commentary was written in an attempt to avoid any repeat of this unfortunate incident that wasted so much of the time and energy of both the journal staff, our reviewers, as well as the authors and their patients.

There is a large volume of material available on what types of research is eligible for waiver of consent. However, the most important point is that the decision to waive consent, written or otherwise, is the sole responsibility of the local IRB/EC. One particularly clear statement about such research comes from the Kansas University School of Medicine Wichita's (KUSM-W) IRB website (wichita.kumc.edu/research/research-compliance.html) which states, 'Guidelines for Retrospective Chart Reviews' are considered to be human subjects research and must be approved by the KUSM-W Human Subjects Committee. Beginning 14 April 2003, human subjects and HIPAA (Health Insurance Portability and Accountability Act) privacy requirements'

Readers who are interested in reviewing requirements for waiver of consent are encouraged to review both local and national consent requirements such as those listed on the US Health and Human Services (HHS) website (http://www.hhs. gov/ohrp/policy/faq/informed-consent/ what-is-a-waiver-or-alteration-of-informedconsent.html). An important quote from the HHS guidelines states, 'Waiving the requirement for obtaining informed consent or parental permission means that the IRB has determined that investigators need not obtain the subjects' informed consent to participate in research' that makes clear that it is the IRB and not the investigator(s) who determines when waiver of consent is allowed. This is also clear in materials from numerous US university IRBs, including retrospective chart reviews must meet both the Northwestern University IRB that states in a section on retrospective chart reviews, 'Research involving the collection or study of existing* data, documents, records, pathological specimens, or diagnostic specimens, if these sources are publicly available or if the information is recorded by the investigator in such a manner that subjects cannot be identified, directly or through identifiers linked to the subjects.

In addition to national or university guidelines/rules, there are a number of local guidelines and these hospital-internal guidelines may impose stricter conditions than required by federal or cantonal law'. Differences and their implications are illustrated by a publication that "provides an overview of the issues for physicians, scientists, ethics committee members and policymakers involved in retrospective research in Switzerland' [1].

In the case of the manuscript submitted to GaBI Journal but then withdrawn, it is highly likely that the two hospital IRBs/ EC's would have approved the study for waiver of consent; provided that the methods used to protect patient confidentiality were judged to be adequate. However, it is the responsibility of these two IRB/ECs and NOT of the authors to decide whether or not this was true.

It is unfortunate that the authors did not seek this approval prior to initiating the study. The study fits with the scope and interests of our journal and we very much wanted to publish it.

We do not know whether the local IRBs/ ECs were asked to approve the study retroactively but refused. Some IRBs (including one that I chaired many years ago) can and do approve such waivers retroactively, but since some do not even consider retroactive approvals investigators are encouraged to always request waiver of consent prior to initiating any such research. This is especially important for authors who wish to publish their research in any journal, including GaBI Journal that requires IRB/ EC review of all human subject research submitted for publication.

"Existing' means existing before the research is proposed to the institutional review board to determine whether the research is exempt (https://irb.northwestern.edu/process/new-study/ reviews/exempt-categories-examples).

DOI: 10.5639/gabij.2016.0501.004

Copyright (c) 2016 Pro Pharma Communications International

Author: Professor Philip D Walson, MD, Editor-in-Chief, GaBI Journal

Submitted: 12 March 2016; Revised: 14 March 2016; Accepted: 14 March 2016; Published online first: 28 March 2016 